\title{
Europäisches Weißbuch zur Allergie aktualisiert
}

\section{Das 1997 erstmals publizierte European Allergy White Paper wurde jetzt aktualisiert. Darin wird der neueste Wissensstand wie der Forschungsbedarf für die Bereiche Epidemiologie, Prävention, Forschung und Patientenorganisationen in einem europäischen Konsens festgehalten.}

eignete Präventionsmaßnahmen einzuleiten - vor allem, um die Entwicklung eines Asthma bronchiale zu verhindern.

Besonderes Augenmerk wird dabei zunächst auf die Innenraumallergene gelegt, deren Bedeutung als Risikofaktor als erwiesen gelten kann. Für die sogenannte „Outdoor-Pollution“ steht der endgültige Beweis für ihre allergiefördernde Wirkung dagegen noch aus, so Wahn. Da aber Erwachsene und Kinder heute bis zu 90\% ihrer Zeit in geschlossenen Räumen verbringen, muß der Innenraumbelastung ohnehin mehr Bedeutung zugemessen werden.

Unabhängig von genetischer Disposition und Umweltfaktoren müssen auch lebensstilbezogene Allergiedeterminanten wie Impfungen, durchgemachte Infektionen oder die Ernährung einer noch geanueren Analyse unterzogen werden. Der ,westliche Lebensstil“" als Risikofaktor kristallisiert sich z.B. beim Vergleich der Asthmaprävalenzen bei Kindern von nur 3\% in Albanien und von 22 bis 40\% in Großbritannien heraus. Für die allergische Rhinitis und das atopische Ekzem wurden ähnlich unterschiedliche Daten erhoben und objektive Parameter wie das spezifische IgE zeigen vergleichbare Verteilungsmuster.

\section{Schwerpunkt Forschung}

Prioritäten im Bereich der Allergieforschung, so St. Holgate (Southampton), liegen auf den Bereichen Prävalenz, Allergieentstehung in der Pränatal- und Säuglingsphase, Sensibilisierungs- und Triggermechanismen sowie der Grundlagenforschung, um die Diagnose und Therapie weiter zu verbessern.

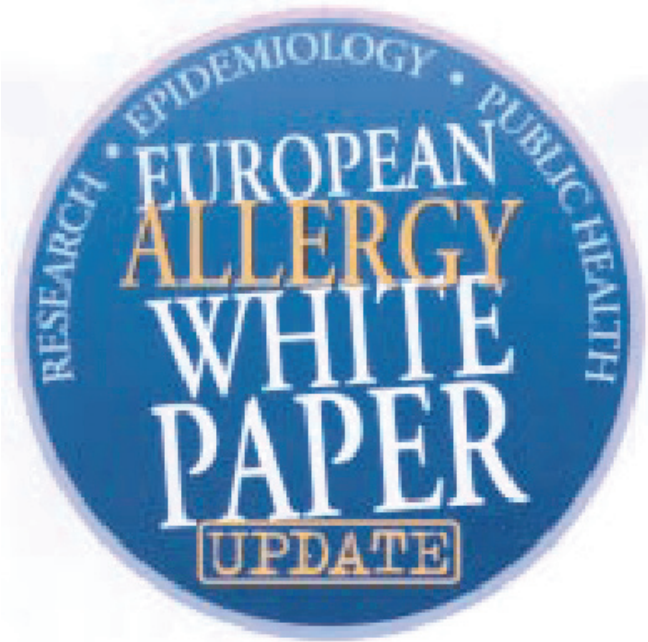

Schwerpunkt Prävention

Wie J. Warner (Southampton) erklärte, ist mit den erarbeiteten Präventionskonzepten bereits ein Fortschritt erzielt worden. Sie basieren auf den wissenschafltich anerkannten Resultaten aus epidemiologischen Untersuchungen wie auch aus der Grundlagenforschung. Warner empfahl gemäß diesem Konzept als Primärprävention bei Risikokindern, also Kindern mit einer positiven Familienanamnese, u.a. über 6 Monate zu stillen, keine Haustiere zu halten, nicht im Beisein der Kinder zu rauchen und die Wohnung gut zu lüften sowie das Kind regelmäßig beim Arzt vorzustellen.

In der Sekundärprävention sollten sensibilisierte Patienten frühzeitig erkannt und vor Allergenen geschützt werden. Allergenkarenz beinhaltet auch hier beispielsweise die Meidung von Haustieren und die Schaffung guter klimatischer Bedingungen in den Wohnräumen. Ferner muß in diesem Stadium die medikamentöse wie nichtmedikamentöse Behandlung optimiert und das Training aller Beteiligten, also von Patienten, medizinischem Personal oder auch von Lehrern verbessert werden.

Die tertiäre Allergieprävention, so Warner, umfaßt die Symptomminimierung durch medikamentöse wie nichtmedikamentöse Therapien, wobei der behandelnde Arzt die eingeleiteten Maßnahmen immer wieder kritisch auf ihre aktuelle Notwendigkeit hin überprüfen sollte.

Satellitensymposium des UCB Institute of Allergy im Rahmen des Annual Meeting of the European Academy of Allergology and Clinical Immunology, Brüssel/Belgien 1999. 